\title{
POLÍTICAS DE ACCESIBILIDAD FÍSICA A LAS CIUDADES UNIVERSITARIAS LATINOAMERICANAS - UNA REVISIÓN CRÍTICA
}

Andrés Borthagaray, Fundación Furban Instituto para la Ciudad en Movimiento. Profesor en posgrados en la Universidad de Buenos Aires (FADU), la Universidad Nacional de Córdoba (FAUD) y la Universidad Politécnica de Cataluña (DUT)

Thomas Massin, Fundación Furban e Instituto Superior de Urbanismo (FADU-UBA)

aborthagaray@gmail.com, tommassin@gmail.com

\section{RESUMEN}

El movimiento reformista significó un cambio en la forma de concebir el rol de la universidad en la sociedad, un espíritu crítico necesario para el desarrollo científico y un movimiento de cambio asociado a una ciudad. Esta idea de movimiento tiene una base intelectual y social, pero se propone analizar aquí la forma en que la movilidad física contribuye a esos objetivos y qué puede aportar el conocimiento a su desarrollo. Para eso estudiamos cuatro casos de ciudades universitarias en Río de Janeiro, Caracas y Buenos Aires. Resulta paradójico que los lugares de desarrollo y transmisión del conocimiento queden atrasados con respecto a las prácticas que deberían guiar un cambio de paradigma por razones de prevención del cambio climático, congestión, inseguridad vial, medio ambiente y salud. Por el contrario, interrogar las prácticas, movilizar a la comunidad universitaria y servirse de su capacidad y conocimiento debería ser un punto de referencia para un cambio en toda la sociedad urbana.

Palabras clave: acceso, movilidad, ciudad, universidad.

\begin{abstract}
A new world of understanding about the way the role of universities are drivers of change, a critical approach in order to develop a true scientific contribution and change associated to a city were key components of the Reform. This idea of movement has clearly an intellectual and social base, but it is proposed to assess here a more literal analysis of its physical roots of mobility and the way knowledge and values are associated to higher education's development. For that we studied the situation of four campus in Rio de Janeiro, Caracas and Buenos Aires. It's paradoxical that places aimed at developing and transmitting development could remain at the rear in terms of practices that should guide a shift in terms of climate change, congestion, road safety, environment and health. In order to change that, it is proposed to question existing practices, mobilize communities and stimulate its capacity and knowledge as a reference for a wider change in urban societies.
\end{abstract}

Keywords: Access, mobility, city, University. 
La Reforma Universitaria que se desencadenó en 1918 en Córdoba, Argentina, significó un cambio en la forma de concebir la universidad, un espíritu crítico necesario para el desarrollo científico y un movimiento de cambio asociado a una ciudad y a una sociedad. Mantener vivo su espíritu, a cien años de aquel movimiento significa poner en cuestión diferentes dogmas con la misma capacidad de renovación del pensamiento. Uno de ellos es el que acepta resignadamente que la universidad se mantenga alejada y aislada de la ciudad, sea difícilmente accesible en transporte público y amigable para la movilidad activa, quede limitada a un uso ineficiente de los recursos de espacio, tiempo, energía y calidad del aire con sus costos en salud y en términos económicos. Resulta paradójico que los lugares de desarrollo y transmisión del conocimiento, aquellos que tienen un rol de vanguardia en estudiar y proponer cambios tanto en la organización social como en los aspectos técnicos de la movilidad, queden atrasados con respecto a las prácticas que deberían guiar un cambio de paradigma por razones de prevención del cambio climático, congestión, inseguridad vial, medio ambiente y salud. Por el contrario, interrogar las prácticas, movilizar a la comunidad universitaria y servirse de su capacidad y conocimiento debería ser un punto de referencia para un cambio en toda la sociedad urbana.

Otro movimiento universitario, cincuenta años después, sacudió a las universidades con antiguas y nuevas reivindicaciones. El 68 del mayo francés tuvo uno de sus primeros episodios en la reivindicación de cuestiones cotidianas en la sede Nanterre, luego ampliada a cuestiones sociales más amplias y convertido en un movimiento nacional. Simbólicamente, la imagen que queda en la memoria es la del Boulevard Saint Michel sin autos (salvo volados o incendiados), marchas y enfrentamientos. El 68 es también el aniversario del movimiento universitario latinoamericano, a partir del movimiento mexicano y de sus trágicas derivaciones en Tlateloco. El 68 es también el año de la publicación del Derecho a la Ciudad de Henri Lefebvre (1968), quien ha publicado también una reflexión sobre Nanterre y el cambio social. Por lo tanto, podemos decir que las reflexiones en la materia tienen como objeto un centenario, pero también un cincuentenario que tiene relación con la cuestión universitaria hispanoamericana.

La historia de la implantación física de las grandes ciudades universitarias de América Latina presenta elementos en los dos sentidos. De vanguardia, en muchos casos vinculada a proyectos modernistas, con referencias que las han convertido en patrimonio universal de la humanidad, como los campus de la Universidad Nacional Autónoma de México (UNAM) -que recientemente celebró sus 60 años- y la Universidad Central de Caracas -también declarada patrimonio de la humanidad ${ }^{1}$, pero también retrógradas en la forma de manejar su accesibilidad en más de un ejemplo. De convivencia con los centros históricos y de desarrollo de nuevas centralidades, más o menos urbanas, más o menos monofuncionales según las situaciones. Así por ejemplo, la UNAM guarda un centro histórico, Bogotá tiene su campus de la universidad nacional en una ubicación excéntrica y un barrio universitario en todo centro, Buenos Aires guarda sedes urbanas y en la ciudad universitaria.

En este artículo, proponemos analizar el acceso a cuatro campus universitarios en tres ciudades de la región: Río de Janeiro, Caracas, Buenos Aires. Hay, por supuesto, muchos otros casos muy interesantes pero aprovechamos para este artículo la existencia de un vínculo muy estrecho con profesores en las tres ciudades estudiadas: Fabiana Izaga en Río de Janeiro, Edgarly Rondon en Caracas y Daniel Kozak en Buenos Aires. Este análisis quiere contribuir a una redefinición de la accesibilidad a y desde las ciudades universitarias a las ciudades. Se basa en un estudio empírico junto a estos tres autores locales y en un estudio teórico que quiere responder a la pregunta: ¿en qué medida el espacio del conocimiento se relaciona con su entorno, crea condiciones para su desarrollo, se ancla en un territorio? Para eso se consideran tres fuentes: un número especial de la revista de Arquitectura de la Universidad de los Andes (DEARQ, 2013), otro de la revista francesa Annales de La Recherche Urbaine (2014) y un sitio internet de estudios especializados en campus (Sasaki, 2018). Más allá de estas referencias específicas, otro eje teórico es el que va entre una perspectiva de unidades monofuncionales, como una generación de planes lo había concebido, y una mixta y polifuncional como, por lo menos en teoría, se tiende a promover en la actualidad. El artículo analiza primero cómo fue su génesis y evolución en los planes urbanos, reflexiona sobre las formas de resolver los problemas en la actualidad y propone una pista de acciones a futuro, desde el análisis de los cuatro casos.

\footnotetext{
${ }^{1}$ Véase http://whc.unesco.org/es/list/1250 y http://whc.unesco.org/es/list/986
} 


\section{EL ACCESO AL ESPACIO DEL CONOCIMIENTO}

El Manifiesto Liminar de la Reforma Universitaria de $1918^{2}$ enunciaba que:

"Las universidades han sido hasta aquí el refugio secular de los mediocres, la renta de los ignorantes, la hospitalización segura de los inválidos y -lo que es peor aún- el lugar en donde todas las formas de tiranizar y de insensibilizar hallaron la cátedra que las dictara. Las universidades han llegado a ser así el fiel reflejo de estas sociedades decadentes que se empeñan_en ofrecer el triste espectáculo de una inmovilidad senil".

Como un elemento de respuesta a esta contundente afirmación del manifiesto liminar se puede destacar el movimiento de generación de nuevas sedes con distintas inspiraciones, que hubo entre las décadas de los 1940 y de los 1960. Los movimientos estudiantiles de la década de los 1960, en particular del mayo 1968 en Francia, pero también en México, Chile y otras ciudades latinoamericanas pusieron en cuestión el rol de las universidades. En un contexto o bien marcado por dictaduras o bien por rasgos fuertemente represivos, lo que había empezado siendo un movimiento de vanguardia terminó siendo un espacio aislado y de contención. Uno de los argumentos es que, a pesar de la vitalidad democrática, algunos de estos elementos no han sido revisados aun con la importancia que se merecen.

Esta debería ser un mero testimonio de la realidad de hace un siglo. Sin embargo, con una mirada crítica a la forma en que los accesos son pensados y concebidos, y aunque se trate más de una inercia cultural que de un acto deliberado, hay margen para cuestionar la forma en que el conocimiento se aplica a las mejores opciones disponibles para una comunidad universitaria en el seno de una sociedad que proyecta en ella expectativas de pensamiento renovado, espíritu crítico y experimentación.

¿Qué rol ocupa la universidad en la sociedad y qué rol ocupa? Es una pregunta a cuya respuesta la celebración de estos aniversarios debiera echar luz. Han tenido grandes debates para elegir a las autoridades, una innovación en la democracia universitaria. Se aspira a que tenga un acceso equitativo en términos sociales, a que sea un canal de movilidad social y de movilidad intelectual.

La idea de acceso, de movilidad -en sentido contrario al "triste espectáculo de una inmovilidad senil" al que alude el manifiesto liminar- es una metáfora física de la realización en todo sentido que tiene ser universitario. La apertura social, de género -con perspectiva del 1918 hasta aquí hay una evolución-, de orígenes diversos. Un ideal de sociedad tiene en la universidad un acceso que no se restringe a los privilegios de la reproducción de las élites (Bourdieu, 2011).

\section{ANÁLISIS DE CUATRO CAMPUS UNIVERSITARIOS}

En general; las ciudades universitarias de universidades públicas se distinguen de las privadas por un sistema más llano y amigable de accesos, menor control policial, aunque progresivamente se han instalado sistemas de seguridad de un tipo u otro, se han cercado donde no lo estaban. Sin embargo, la llegada física por un lado y socioeconómica por otro no es tan fácil.

\section{Universidad de Buenos Aires (UBA) (Kozak, 2018)}

El caso de la Ciudad Universitaria de la UBA puede servir como un ejemplo de los problemas que tienen las ciudades universitarias en América Latina de sus débiles vínculos con las ciudades que las albergan. Por las características de su población, por el potencial paisajístico y la cercanía con algunos de los mejores circuitos de Buenos Aires, podría ser uno de los principales generadores de viajes en modos no-motorizados de la ciudad. Sin embargo, quienes la frecuentan, conocen los problemas de accesibilidad a la Ciudad Universitaria, que suelen tornarse notablemente más agudos y graves para ciclistas y peatones.

El único acceso para modos no-motorizados en el sur del predio -desde donde llega la mayoría de estudiantes, docentes y demás trabajadores - resulta insuficiente e inadecuado desde todo punto de vista: es inseguro e inhóspito, y está alejado de los pabellones II y III, en donde se concentra la mayor parte de la población de la CU. Se trata del puente Scalabrini Ortiz, emplazado sobre donde hasta hace poco funcionaba la estación homónima del ferrocarril Belgrano Norte, recientemente clausurada y reemplazada por la nueva estación Ciudad Universitaria. Si bien la nueva estación mejoró notablemente la llegada de transporte público,

\footnotetext{
2 Disponible en https://www.unc.edu.ar/sobre-la-unc/manifiesto-liminar
} 
se ha perdido una preciada oportunidad para fortalecer el vínculo con la ciudad. La nueva estación conforma un puente sobre las vías del tren y Cantilo, y -absurdamente- no cruza Lugones. Si lo hiciera, podría conectarse con la continuación de la calle Sáenz Valiente -cedida por el Gobierno de la Ciudad de Buenos Aires al Club River Plate-, y así con la ciclovía de Figueroa Alcorta. Este sería un primer paso para acercar la CU a la ciudad y transformarla en uno de los lugares más atractivos e interesantes que podría tener Buenos Aires, a partir de sumar nuevos usos, más allá de su actual monofuncionalidad, y la evidente subutilización de su ubicación favorecida

\section{Universidad Central de Venezuela (UCV) y Universidad Simón Bolívar (USB) en Caracas (Rondon, 2018)}

La UCV fue diseñada en 1944, en los terrenos de la antigua Hacienda Ibarra debido a las condiciones topográficas y su ubicación en el corazón de la ciudad. El diseño arquitectónico contempla el reconocimiento y las cualidades de los distintos espacios para generar una armonía entre sus usuarios y las condiciones climáticas. Por el contrario, la USB, es inaugurada en 1970 por una necesidad gubernamental, con un proyecto muy poco cautivador. Su diseño solo juega con la réplica de estructuras y de algunas variaciones en la forma según el uso, aunque se destaque el diseño de sus jardines. Su ubicación en el sureste de la ciudad y en un relieve montañoso no permite la integración con el resto de la Ciudad de Caracas.

En lo que refiere a la accesibilidad, la UCV es privilegiada: cuenta con la estación Ciudad Universitaria del Metro de Caracas, dos rutas de transporte público superficial e intermunicipal que transitan dentro del campus con excelente conectividad con las principales arterias viales de la ciudad, lo que permite acceder a la misma en vehículo particular, de alquiler, transporte universitario, en bicicleta y de forma peatonal. La movilidad dentro de la UCV, prioriza tanto el uso del vehículo particular como la movilidad peatonal y permite el desplazamiento de personas con movilidad reducida, ya que fue diseñada para ello.

En cambio, la accesibilidad a la USB es restringida, su ubicación privilegia el uso del vehículo particular y del transporte universitario que cubre rutas intermunicipales y suburbanas, y cuenta con una ruta de transporte público urbana que no es permeable al campus. La movilidad interna prioriza los desplazamientos peatonales, pero restringe los de las personas con movilidad reducida y las condiciones topográficas hacen más atractivo el modo vehicular para recorridos superiores a los 500 metros. El privilegio del uso del vehículo automotor para la movilidad, no solo responde a la distancia de los desplazamientos, sino a las consecuencias de políticas públicas que incentivan el consumo de combustible fósil. Dicha política, se respalda en el costo absurdo del combustible -no representa ni un centavo de dólar por litro y el abastecimiento de un vehículo es menor que la tarifa mínima de transporte público, aunado a una disminución en la oferta de transporte público - debido al desabastecimiento y alto costo de los repuestos- $y$ al retraso de los proyectos de transporte masivo. Sin embargo, existen proyectos que buscan evolucionar a medios más sostenibles para hacer las ciudades universitarias más accesibles e inclusivas, como "Ruédala Simón" (iniciativa promociona el uso de la bicicleta y la creación de ciclo caminerías dentro de la USB), los trabajos del Centro de Estudio de Movilidad en Bicicleta (CEMBI), varias iniciativas para promover el uso de la bicicleta y proyectos como "\#MOVER" y "Accesibilidad Universal" destinado a la Ciudad de Caracas y sus universidades, buscan la cohesión y la integración social, la adecuación de infraestructura y de medios de transporte para personas con movilidad reducida, especialmente las que residen en asentamientos informales.

\section{Universidade Federal do Rio de Janeiro (UFRJ) (Izaga y Silveira, 2018)}

En Río de Janeiro, la ciudad universitaria de la UFRJ está localizada en el gran aterro sobre las aguas de la Bahía de Guanabara que reúne 8 islas, entre ellas la Isla de Fundão, en un área que abarca 5,2 kilómetros cuadrados. Con una población fluctuante de 60 mil personas por día, la Isla de Fundão es también un local de residencia permanente para 1556 personas que viven en la Villa Residencial, asentamiento originariamente informal, hoy regularizado, al que se suman 300 estudiantes, que ocupan dos edificios de residencia estudiantil.

El grandioso plano general del campus de 1955 -que hasta el día de hoy posee extensas áreas desocupadases tributario de los principios del movimiento moderno de matriz corbusierana, donde la circulación vial y estructurante está en consonancia con la idea de edificios sueltos, cuyo plano de fondo es el verde de un parque. Uno de los mayores problemas que la ciudad enfrenta en este siglo XXI es el de la movilidad urbana, en vista de la dificultad de acceso a la Isla do Fundão, reducida a tres conexiones viales por medio de vías expresas. Siguiendo la tendencia de la matriz modal de la ciudad, los ómnibus son el transporte predominante con casi el $50 \%$ de los viajes, con un $20 \%$ para el auto particular. 
Ni los ferrocarriles ni el metro llegan a la Isla, por lo que se necesitan de ómnibus de media distancia para poder alcanzarla. Con esto, la isla posee aproximadamente $238000 \mathrm{~m} 2$ de estacionamientos, dispersos en los entornos de los principales edificios. El campus cuenta todavía con una terminal de ómnibus en su extremo norte, que recibe al BRT Transcarioca y líneas municipales e intermunicipales de alcance metropolitano. A su vez un ómnibus interno se ofrece gratuitamente con tres itinerarios circulares que funcionan las 24 horas al día los siete días a la semana.

En el contexto de una política de bajo carbono, la UFRJ viene desarrollando iniciativas de investigación y asociaciones para la promoción de la movilidad sustentable. Por ejemplo, la Oficina de Proyectos del Fondo Verde para el Desarrollo de Energía para la Ciudad Universitaria de Río de Janeiro fue instituido en 2013 por medio de decreto estatal. La institución recibe recursos de la tasa de exención del impuesto cobrado por el gobierno de Río de Janeiro sobre la cuenta de luz del campus de la Ciudad Universitaria, con el fin de invertir y mejorar los sectores de movilidad, eficiencia energética, reducción de consumo de agua y residuos, monitoreo de datos y generación de indicadores en el campus.

El programa para la movilidad intracampus, Programa de Movilidad Fondo Verde-UFRJ", apoya proyectos orientados a la movilidad sustentable, los cuales engloban minibuses que realizan trayectos internos y una propuesta de carsharing, con diez puntos de detención alineados con los principales centros de la Ciudad Universitaria. Todavía se estudia la posibilidad de implementación de un sistema compartido de bicicletas con auspiciante, como el que ya está implantado en la zona sur de la ciudad, pero que no se concreta debido a la previsión de poca demanda para la articulación interna con otros modos.

Finalmente, aunque todas estas iniciativas sean loables para la promoción de una actualización tecnológica y de innovación de alternativas energéticas para una movilidad sustentable, el Campus de la isla do Fundão se resiente desde el punto de vista urbano, con sus gigantescas dimensiones internas y de la exagerada escala de su conjunto en relación a la ciudad. Aún con los direccionamientos recientes de tratamiento de sus vacíos, con la investigación de polos avanzados de investigación y tecnología, nos parece que la dimensión urbana tenderá a complicarse si no existe una política para aumentar la población residente. Las iniciativas para una movilidad urbana de bajo carbono no podrían ser pensadas por fuera del diseño de los espacios públicos y de convivencia, sino que deben estar integradas a las políticas que contemplen la vida urbana en toda su complejidad.

\begin{tabular}{|c|c|c|c|}
\hline Ejemplos & Sedes & Año / Ubicación & $\begin{array}{l}\text { Sistema de accesibilidad } \\
\text { CU }\end{array}$ \\
\hline $\begin{array}{l}\text { Buenos Aires: } \\
\text { Universidad de Buenos } \\
\text { Aires (UBA) }\end{array}$ & $\begin{array}{l}13 \text { facultades y } 9 \text { sedes } \\
\text { La Ciudad Universitaria es } \\
\text { sede de la Facultad de } \\
\text { Arquitectura, Diseño y } \\
\text { Urbanismo y de la Facultad } \\
\text { de Ciencias Exactas y } \\
\text { Naturales. También es una } \\
\text { de las sedes del Ciclo } \\
\text { Básico Común y es el Área } \\
\text { deportiva de la Universidad. }\end{array}$ & $\begin{array}{l}\text { Proyecto de la década de } \\
\text { los } 1950 \\
\text { Ejecución en la década de } \\
\text { los } 1960 \\
\text { Ubicación en la Ciudad } \\
\text { Autónoma de Buenos Aires } \\
\text { pero excéntrica }\end{array}$ & $\begin{array}{l}\text { Sobre todo: automóvil, } \\
\text { colectivo, tren } \\
\text { Difícil acceso mediante } \\
\text { modos no motorizados. } \\
\text { Proyectos formulados de } \\
\text { promoción, concursos } \\
\text { nacionales de ideas para } \\
\text { estudiantes demostrativos } \\
\text { de alternativas. }\end{array}$ \\
\hline $\begin{array}{l}\text { Caracas: } \\
\text { Universidad Central de } \\
\text { Venezuela }\end{array}$ & $\begin{array}{l}11 \text { facultades } \\
\text { La sede principal es Ciudad } \\
\text { Universitaria de Caracas }\end{array}$ & $\begin{array}{lll}\text { Diseñada en } 1944 \text { (hoy } \\
\text { patrimonio histórico de la } \\
\text { humanidad para } & \text { la } \\
\text { UNESCO) } & & \\
\text { Céntrica } & & \end{array}$ & $\begin{array}{l}\text { Estación Ciudad } \\
\text { Universitaria del Metro de } \\
\text { Caracas } \\
\text { Dos rutas de transporte } \\
\text { público superficial e } \\
\text { intermunicipal que transitan } \\
\text { dentro del campus con } \\
\text { excelente conectividad con } \\
\text { las principales arterias } \\
\text { viales, lo que permite } \\
\text { acceder a la misma en } \\
\text { vehículo particular, de }\end{array}$ \\
\hline
\end{tabular}




\begin{tabular}{|c|c|c|c|}
\hline & & & $\begin{array}{l}\text { alquiler, transporte } \\
\text { universitario, en bicicleta y } \\
\text { de forma peatonal. }\end{array}$ \\
\hline Universidad Simón Bolívar & $\begin{array}{l}2 \text { sedes } \\
4 \text { grandes áreas de } \\
\text { disciplinas universitarias }\end{array}$ & $\begin{array}{l}\text { Inaugurada en } 1970 \\
\text { Excéntrica }\end{array}$ & $\begin{array}{l}\text { Se privilegia el uso del } \\
\text { vehículo particular y del } \\
\text { transporte universitario que } \\
\text { cubre rutas } \\
\text { intermunicipales y } \\
\text { suburbanas, y cuenta con } \\
\text { una ruta de transporte } \\
\text { público urbana que no es } \\
\text { permeable al campus. }\end{array}$ \\
\hline $\begin{array}{l}\text { Río de Janeiro: } \\
\text { Universidade Federal do } \\
\text { Rio de Janeiro (UFRJ) }\end{array}$ & $\begin{array}{l}3 \text { sedes, unas seis } \\
\text { unidades universitarias } \\
\text { La sede principal es el } \\
\text { Campus Universitario en } \\
\text { Isla de Fundão }\end{array}$ & $\begin{array}{l}\text { Plano general del campus } \\
\text { de } 1955 \\
\text { Si bien se desarrolla en un } \\
\text { área céntrica de la ciudad, } \\
\text { generó una isla urbana, que } \\
\text { cuenta con sólo } 3 \text { puentes } \\
\text { de acceso. }\end{array}$ & $\begin{array}{l}\text { Ómnibus: } 50 \% \text { de los viajes } \\
\text { Auto particular: } 20 \% \text { de los } \\
\text { viajes } \\
\text { Sin metro y sin tren } \\
\text { Sistema de ómnibus interno } \\
\begin{array}{l}\text { Buen sistema interno de } \\
\text { bicicletas }\end{array}\end{array}$ \\
\hline
\end{tabular}

Tabla de comparación en tres ciudades. Fuentes: Kozak (2018), Rondon (2018), Izaga y Silveira (2018).

Sería poco riguroso un análisis que pretenda generalizar casos a partir de realidades muy diversas en América Latina. Por otra parte, la muestra que integra estos ejemplos está lejos de ser representativa. Los recortes socio económicos son muy distintos de acuerdo al modelo organizativo de las universidades en cuestión.

En síntesis, reconsiderar la política de accesos y desplazamiento puede tener un efecto importante tanto en el acceso como en la salud de la comunidad universitaria, en su relación con la comunidad más amplia a la que pertenece, con beneficios sociales, ambientales, físicos y económicos. En un marco más grande, el rol de las universidades es fundamental para las ciudades. Lo demuestra una investigación realizada a partir de una base de más de 15000 universidades en 1500 regiones en 78 países. La presencia de universidades es un factor de dinamismo asociado al desarrollo (se estima que duplicar el número de universidades per cápita implica un aumento de 4\% del PIB per cápita), a partir de factores directos e indirectos, así como una correlación con el nivel de intensidad democrática (Valero y Van Reenen, 2016; Florida, 2017). Dicho de otra forma, a medida que aumenta la densidad de universidades, mayor es el dinamismo económico. Así, las universidades no son sólo un reflejo del desarrollo sino una de sus causas.

\section{3. ¿CÓMO MEJORAR? PAUTAS PARA EL MANIFIESTO DE UN TIPO DE ACCESO A CIEN AÑOS DE LA REFORMA, QUE ROMPA LA "INMOVILIDAD SENIL"}

Un análisis somero de casos puntuales y una encuesta preliminar ampliada nos muestran un momento de cambio en la forma de concebir los accesos a las ciudades universitarias latinoamericanas y sus articulaciones con los respectivos centros urbanos. Si bien las sedes tradicionales de las universidades han tendido a quedar chicas para sus demandas crecientes de espacio, el rol que juegan en la vitalidad de los centros sigue siendo clave, multiplicado además por una combinación con nuevas universidades que consideran atractivos los entornos centrales. Por otra parte, en aquellos casos en los que la extensión de las ciudades universitarias lo demanda, se han tendido a desarrollar sistemas internos de articulación por dispositivos de alquiler de bicicletas (como en la UFRJ, la UNC de Bogotá, la Universidad Nacional de Córdoba). En otras situaciones, como la de la Ciudad Universitaria de la UBA, a pesar de que hay una serie de iniciativas en la ciudad, todavía no se ha desarrollado un dispositivo interno. Pero globalmente, los desafíos de articulación mediante las redes de transporte público siguen pendientes. Por supuesto, hay una enorme diversidad de casos. En la Ciudad Universitaria de la UNAM hay tres estaciones de metro y en San Pablo una línea nueva se acercó a una distancia accesible. 
Pero igualmente importante es la integración urbana. En ese sentido, el Proyecto "Transformación Urbana: Ciudad Universitaria Rosario", presentado por la Municipalidad en febrero 2018, tiene como objetivo el reordenamiento urbano del área del Centro Universitario (CUR) e incluye la construcción de la Avenida de la Universidad para vincular e integrar el CUR con el resto de la ciudad y de 321 nuevas viviendas que solucionarán el problema de los asentamientos irregulares sobre terrenos de la Universidad. Además, se recuperará la barranca con el fin de revalorizar el sector, se ejecutarán mejores accesos viales, una nueva estructura de movilidad, nuevos espacios públicos y se pondrá en valor los existentes.

Desde otro ángulo, el espacio y el acceso tienen ejes de cambio alternativos. Por un lado, la accesibilidad por medios no motorizados requiere de espacios seguros, confortables y estimulantes. Por otro, las políticas frente al cambio climático y en pos de los parámetros más exigentes de salud, a partir de la evidencia sobre el efecto de la calidad del aire, modifican la forma de concebir estas articulaciones: existe una evidencia incontrastable de enfermedades y muertes jóvenes que se pueden prevenir, tanto por cuestiones de seguridad vial como de hábitos sedentarios y su efecto en la obesidad, diabetes y otras patologías como por los efectos del aire que se respira en la salud (Silverman et al., 2012).

La propia forma de utilización del espacio del conocimiento alterna formas tradicionales -como las aulas y salas de conferencias, donde lo que evoluciona sobre todo son los equipos de sonido y proyección, pero no tanto el espacio físico- con cambios producidos por la digitalización de los espacios de lectura e interacción. Un conjunto de grandes universidades ha abierto cursos gratuitos on line, como gesto de apertura y, en parte, como parte de la imagen institucional. Ahora bien, queda pendiente un reflejo equivalente desde el espacio: ¿en qué medida se abren las universidades a la comunidad? La cuestión del acceso a la universidad tiene un sentido literal y uno más amplio. Uno se reduce a la forma de llegar a las sedes, otro al estudio universitario e, idealmente, a un grado profesional que permita promover la movilidad social. Claro que para ser efectiva, las formas de mejora deben tener múltiples canales y no un embudo en la universidad. Tanto en el sentido literal como en el metafórico.

En ese sentido, se ha cumplido un ciclo. En las décadas posteriores a 1918 se desarrollaba una visión urbana, que tendría su mayor desarrollo en América Latina poco después de promediar el siglo, según la cual era más eficiente separar las funciones, cuyo producto eran unidades monofuncionales. Si bien la discusión desde el punto de vista de la teoría urbana es más compleja y contradictoria, varios de los proyectos de ciudades universitarias han seguido esta lógica. Hoy se ha revalorizado la mixidad, pero todavía no ha alcanzado, salvo excepciones, a las ciudades universitarias. El emplazamiento de las universidades y su relación con el entorno es un punto importante de la nueva generación de planes urbanos que se desarrolle de aquí en más. La idea sagrada de la autonomía no debe confundirse con la insularidad.

Retomando el razonamiento inicial, a pesar de grandes mejoras en general, y con grandes altibajos producto de una historia conflictiva, con rupturas en el orden democrático en distintos momentos de la historia, la "inmovilidad senil" a la que hacía referencia el manifiesto liminar de la Reforma sigue vigente en ciertas formas de concebir los desplazamientos. Lo que hace cien años apenas se insinuaba hoy ya empieza a ser obsoleto: la dependencia en vehículos de combustión interna y el espacio funcional a su utilización como medio primario para quienes pueden disponer de él. "Llamar a las cosas por su nombre" en materia de acceso a las ciudades universitarias requiere de aportes críticos desde conocimientos urbanísticos, de ingeniería, arquitectura y de observación de los modos de vida; ambientales, por los niveles de emisión y la contribución de gases de efecto invernadero, a cuya disminución los países que tratamos aquí se han comprometido.

A pesar de buenas intenciones en el origen y en algunas medidas puntuales en la actualidad, queda pendiente que el espíritu crítico que se celebra en el movimiento reformista se refleje en las políticas actuales de acceso, en el espacio de las ciudades universitarias y en sus múltiples articulaciones con la ciudad de la que forman parte y de cuyo desarrollo son una parte esencial. Los pequeños pasos que se han dado todavía no terminan de marcar un rumbo. 


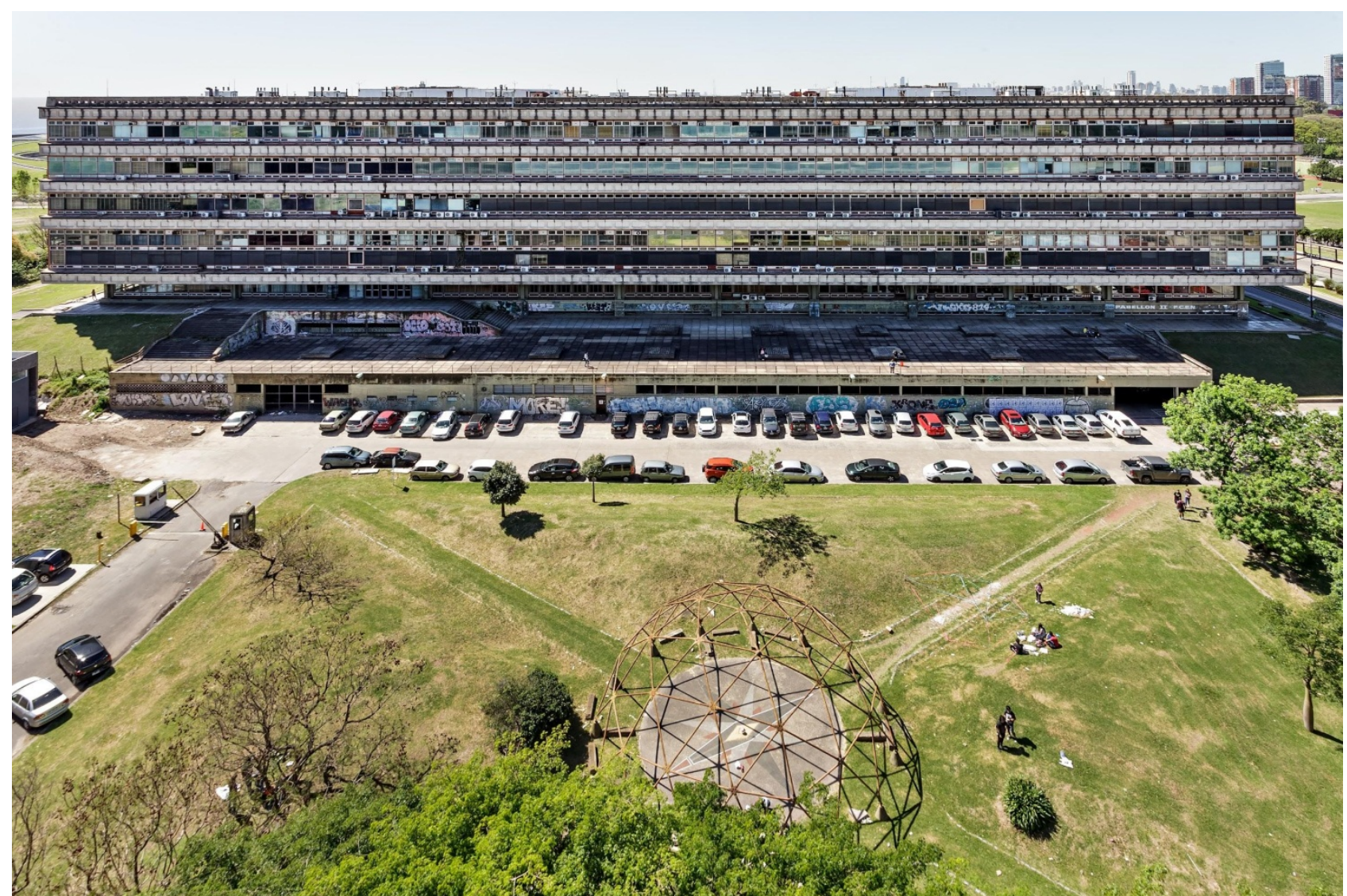

Pabellón 2 visto desde el pabellón 3, Ciudad Universitaria de Buenos Aires. Fotografía de Albano García (investigación fotográfica para la revista Arquis)

\section{BIBLIOGRAFÍA PRINCIPAL}

ARQUIS. (2018). El espacio de la movilidad post - fósil. Universidad de Palermo (Buenos Aires), 8

BOURDIEU, P. (2011). Las Estrategias de la Reproducción social. Siglo XXI, Buenos Aires

DEARQ (2013). Revista de Arquitectura / Journal of Architecture, Universidad de Los Andes (Bogotá), 13. https://issuu.com/dearq/docs/dearq 13 universidades y territorio

Electric Car: the death of the internal combustion engine. It had a good run. But the end is in sight for the machine that changed the world. (2017, 12 de agosto). The Economist, Print edition: leaders

FLORIDA, R. (2017). How Universities Foster Economic Growth-and Democracy. Citylab. https://www.citylab.com/equity/2017/11/how-universities-foster-economic-growthanddemocracy/545051/?utm source=nl link6 110717\&silverid=MzgxMjM3NDY5NDQ3S0

IZAGA, F. y SILVEIRA, A. (2018). Universidad Federal de Río de Janeiro. Un gigante en busca de la movilidad sustentable. Revista ARQUIS, Universidad de Palermo (Buenos Aires), 8

KOZAK, D. (2018). Acercar Ciudad Universitaria a la ciudad. Revista ARQUIS, Universidad de Palermo (Buenos Aires), 8

LEFEBVRE, H. (1968). Le droit a la ville. Paris. Anthropos, 1968

LES ANNALES DE LA RECHERCHE URBAINE. (2014). Territoires et universités. PUCA (París), 105

REVISTA R. (2014). Passages, Espacios de Transición del Siglo XXI. FADU-UDELAR (Montevideo). 
RONDON, E. (2018). Universidad Central de Venezuela / Universidad Simón Bolívar Dos casos emblemáticos en Caracas. Revista ARQUIS, Universidad de Palermo (Buenos Aires), 8

SASAKI. (2018). Sitio internet: http://www.sasaki.com/blog/view/1032/

SILVERMAN, D et al. (2012).The Diesel Exhaust in Miners Study: A Nested Case-Control Study of Lung Cancer and Diesel Exhaust. Journal of the National Cancer Institute 104, no. 11, 855-68

VALERO, A. y VAN REENEN, J. (2016). The Economic Impact of Universities: Evidence from Across the Globe". CEP Discussion Paper, 1444 